\title{
Les municipalites et le defi interculturel : l'apport des communications
}

\section{Caroline Andrew et Louise Legault}

\section{(2) OpenEdition \\ 12 Journals}

Édition électronique

URL : http://journals.openedition.org/communicationorganisation/1730

DOI : 10.4000/communicationorganisation. 1730

ISSN : $1775-3546$

Éditeur

Presses universitaires de Bordeaux

Édition imprimée

Date de publication : 1 novembre 1994

ISSN : 1168-5549

\section{Référence électronique}

Caroline Andrew et Louise Legault, « Les municipalites et le defi interculturel : l'apport des communications », Communication et organisation [En ligne], 6| 1994, mis en ligne le 26 mars 2012, consulté le 19 avril 2019. URL : http://journals.openedition.org/communicationorganisation/1730 ; DOI : 10.4000/communicationorganisation. 1730

Ce document a été généré automatiquement le 19 avril 2019

(c) Presses universitaires de Bordeaux 


\title{
Les municipalites et le defi interculturel : l'apport des communications
}

\author{
Caroline Andrew et Louise Legault
}

1 Les municipalités québécoises commencent à réfléchir sur leurs activités dans le domaine interculturel. Lentement, les gouvernements locaux se rendent compte de la diversité grandissante de leurs populations. Dans ce texte, nous voulons étudier la création de politiques locales, en regardant le rôle joué par les communications dans cette prise de conscience. Notre réflexion sera fondée sur l'étude d'une expérience de recherche-action effectuée par le Conseil interculturel de l'Outaouais auprès des trois plus grandes municipalités de l'Outaouais québécois ; Hull, Gatineau, Aylmer.

2 Les communications ont joué un rôle central dans cette recherche-action et dans le processus de réflexion sur les politiques appropriées au domaine interculturel. L'objectif de ce texte est de bien comprendre et d'expliquer le rôle crucial des communications dans cette recherche-action.

3 Avant d'aborder notre étude de cas, nous situerons le contexte de l'analyse en fournissant certains éléments sur le système municipal québécois, sur la région de l'Outaouais et en particulier sur les trois municipalités étudiées, et sur la politique de régionalisation de l'immigration du Gouvernement du Québec.

\section{Le système municipal québécois}

4 Il importe de brosser un tableau rapide du système municipal québécois pour pouvoir souligner à quel point la préoccupation pour le domaine interculturel représente un changement par rapport à l'activité «traditionnelle» des municipalités. Les gouvernements locaux au Québec concentrent leurs activités sur la gestion de la propriété privée; les aqueducs et égouts, les routes, la police et les pompiers, l'aménagement et le zonage. Les services aux individus ne relèvent que très peu des municipalités ; en général leur responsabilité revient au Gouvernement du Québec. Selon 
les dirigeants municipaux, le partage des responsabilités est clair; le gouvernement provincial doit s'occuper des politiques sociales et des questions concernant la redistribution des revenus. Ce point de vue s'appuie sur un argument fiscal, qu'il est illogique et inapproprié d'utiliser une source de taxation comme l'impôt foncier (la principale source autonome des revenus municipaux) dont l'impact est régressif pour pouvoir effectuer des dépenses qui visent la redistribution. Selon les élus municipaux, un tel système équivaut à taxer les pauvres pour tenter de les rendre moins pauvres. Nous n'allons pas débattre de cette question de la véritable nature de l'impôt foncier qui ne fait d'ailleurs pas l'unanimité chez les économistes. Nous voulons plutôt souligner la nature du discours des municipalités qui tente de justifier la division actuelle des responsabilités et l'activité très minime des gouvernements locaux dans le secteur des politiques sociales.

5 En plus d'être dominées par les activités qui visent les services à la propriété, les municipalités sont très dépendantes du gouvernement québécois. Le contrôle du gouvernement s'exerce beaucoup sur le plan financier, car une partie importante des budgets municipaux provient des subventions du Gouvernement du Québec. La dépendance a une dimension légale - les municipalités peuvent être considérées comme les créateurs du gouvernement du Québec - mais elle est beaucoup motivée par la prudence fiscale des gouvernements locaux.

6 Le contexte spécifique des relations entre le Gouvernement du Québec et les municipalités au moment de la recherche-action ajoute également à la réticence des municipalités d'assumer de nouveaux domaines d'activité. Le Gouvernement du Québec vivait une phase de décentralisation des activités, tout en ayant manifesté sa volonté d'obliger les gouvernements municipaux à accepter un fardeau financier accru (Ministère des affaires municipales, 1990). Dans les discussions sur le partage des responsabilités, le gouvernement du Québec avait indiqué que ses actions représentaient un début et certainement pas la fin des activités de décentralisation. Les municipalités avaient donc de bonnes raisons de craindre que leur situation budgétaire allait s'empirer, une perception qui ajoutait à leur prudence habituelle.

\section{Les municipalités urbaines de l'Outaouais québécois}

7 La caractéristique principale des trois municipalités de notre étude; Hull, Gatineau et Aylmer est leur double allégeance. Elles sont des municipalités de taille moyenne du réseau municipal québécois et, en même temps, elles font partie intégrante de la région métropolitaine d'Ottawa-Hull, donc de la quatrième plus grande agglomération urbaine du Canada - après celles de Toronto, Montréal et Vancouver.

Mais, tout en faisant partie d'une grande agglomération urbaine, les trois municipalités sont de taille moyenne. En 1986 les populations étaient les suivantes: Gatineau 81,200; Hull 58,700; et Aylmer 29,000. Les administrations municipales reflètent cette réalité démographique - les bureaucraties locales sont relativement simples et il y a relativement peu de personnel professionnel. Il y a également un niveau régional de gouvernement qui chapeaute les municipalités du côté québécois mais ce palier intervient encore moins dans le domaine social que les gouvernements locaux. Il s'occupe plutôt des questions de financement et de fonctionnement des grandes infrastructures.

9 Ce double caractère revient continuellement dans notre analyse de l'Outaouais québécois. La réalité sociodémographique est celle d'une grande agglomération métropolitaine mais 
les structures municipales qui gèrent cette réalité sont les structures municipales typiques des villes moyennes.

Pour compléter ce portrait régional, regardons brièvement la distribution de la population immigrante. En 1986, environ 5\% de la population régionale était considérée comme immigrante. Pour nos trois municipalités, les chiffres sont les suivants : Hull 3515 personnes, Gatineau 2300 et Aylmer 2210. La communauté immigrante la plus importante dans la région est la communauté portugaise, centrée à Hull. Plus récemment, pour la période 1987-1991, le tableau 1 indique les pays d'origine des immigrants qui eurent comme destination l'Outaouais. Les deux pays qui viennent en tête de liste sont le Liban et le Vietnam.

Tableau 1 - Répartition des immigrants admis au Québec selon les principaux pays de dernière résidence, pour la région de destination de l'Outaouais, 1987-1991. (Données préliminaires pour 1991)

Source : Direction des études et de la recherche, Ministère des communautés culturelles et de l'immigration du Québec

\begin{tabular}{|c|c|c|c|c|c|c|c|}
\hline \multirow{2}{*}{$\begin{array}{c}\text { Principaux } \\
\text { pays de } \\
\text { dernière } \\
\text { résidence }\end{array}$} & 1987 & 1988 & 1989 & 1990 & \multirow{2}{*}{$\begin{array}{r}1991^{*} \\
213\end{array}$} & \multicolumn{2}{|c|}{$\begin{array}{l}1987-1991 \\
\text { Nombre \% }\end{array}$} \\
\hline & 58 & 28 & 135 & 210 & & 644 & \\
\hline Roumanie & 57 & 104 & 164 & 175 & 97 & 597 & 17,4 \\
\hline Portugal & 14 & 26 & 37 & 55 & 41 & 173 & 5,0 \\
\hline Kampuchéa & 5 & 18 & 41 & 68 & 39 & 171 & 5,0 \\
\hline Pologne & 25 & 34 & 69 & 12 & 4 & 144 & 4,2 \\
\hline France & 32 & 24 & 36 & 29 & 12 & 133 & 3,9 \\
\hline $\begin{array}{l}\text { El Salvador } \\
\text { F́tats-Inis }\end{array}$ & 17 & 7 & 15 & 28 & 28 & 95 & 2,8 \\
\hline $\begin{array}{l}\text { Etats-Unis } \\
\text { Haiti }\end{array}$ & 16 & 16 & 27 & 19 & 17 & 95 & 2,8 \\
\hline $\begin{array}{l}\text { Haiti } \\
\text { Etthiopie }\end{array}$ & $\begin{array}{l}13 \\
14\end{array}$ & $\begin{array}{l}8 \\
8\end{array}$ & ${ }_{25}^{22}$ & $\begin{array}{l}16 \\
15\end{array}$ & 28 & 87 & 2,5 \\
\hline $\begin{array}{l}\text { Ethiopie } \\
\text { Iran }\end{array}$ & $\begin{array}{c}14 \\
5\end{array}$ & 28 & 11 & $\begin{array}{l}15 \\
23\end{array}$ & 21 & 83 & 2,4 \\
\hline Hongrie & 3 & 29 & 13 & 7 & ${ }_{13}^{6}$ & 73 & 2,1 \\
\hline Mexique & 0 & 10 & 43 & 9 & $\begin{array}{c}13 \\
0\end{array}$ & 65 & 1,9 \\
\hline Laos & 9 & 4 & 4 & 17 & 27 & 62 & 1,8 \\
\hline Hong Kong & 0 & 0 & 3 & 5 & 49 & $\begin{array}{l}61 \\
57\end{array}$ & $\begin{array}{l}1,8 \\
1,7\end{array}$ \\
\hline Somalie & 15 & 7 & 10 & 3 & 9 & 44 & 1,3 \\
\hline Maroc & 0 & 2 & 5 & 11 & 18 & 36 & 1,1 \\
\hline Pérou & 5 & 5 & 4 & 3 & 18 & 35 & 1,0 \\
\hline Thécosbraquie & 4 & 2 & 5 & 5 & 17 & 33 & 1,0 \\
\hline Autres pays & 5 & 0 & 17 & 9 & 2 & 33 & 1,0 \\
\hline TOTAL & 79 & 150 & 158 & 156 & 164 & 707 & 20,6 \\
\hline & 376 & 510 & 844 & 875 & 823 & 3428 & 100 \\
\hline
\end{tabular}

11 Les municipalités et le défi...

\section{La régionalisation de l'immigration - les politiques du gouvernement du Québec}

Le dernier aspect qu'il convient de présenter pour notre étude a trait aux politiques du Gouvernement du Québec au sujet de la régionalisation de l'immigration. Notre intention ici n'est pas de faire une étude de ces politiques (pour des analyses, voir Bonneau et Tremblay, 1993; Folco 1993, Secrétariat régional, 1992) mais plutôt d'indiquer leur importance pour la compréhension de notre étude de cas. Les municipalités savent que le gouvernement veut favoriser les activités qui encouragent les immigrants de s'établir en région. Étant donnés les rapports de dépendance des municipalités à l'égard du Gouvernement du Québec que nous avons décrit plus tôt, les municipalités cherchent des occasions d'être bien perçues par les autorités provinciales. Le domaine de l'immigration semblait offrir de telles possibilités. 

action effectuée dans la partie urbaine de l'Outaouais québécois qui visait à augmenter l'activité municipale dans le domaine des relations interculturelles. Notre but est de comprendre de quelle façon et pourquoi les communications ont été au centre de cette intervention. e-s des communautés culturelles qui a pris l'initiative de l'étude. Le Conseil a reçu une subvention du Ministère des communautés culturelles et de l'immigration du Québec pour un projet visant à sensibiliser les municipalités à la réalité interculturelle. Certains membres du Conseil avaient travaillé à la réalisation du colloque sur la régionalisation de l'immigration qui avait eu lieu dans la région en novembre 1991 et le projet de rechercheaction s'inscrivait dans les suites du colloque. Les membres du Conseil, sachant l'intérêt du ministère pour la régionalisation, ont formulé un projet qui allait dans le même sens que la vision ministérielle. La subvention a été accordée au Conseil et cette décision est déjà une illustration de l'importance politique des liens de communication. II est presque impossible de décider si une telle subvention représente plus une initiative gouvernementale qu'une initiative de la société civile. On peut l'interpréter comme le Ministère qui bénéficie de l'insertion régionale du Conseil ou comme le Conseil qui bénéficie de l'argent du Ministère mais ce qui ressort de toutes les interprétations, c'est l'importance des communications entre le Ministère et le Conseil interculturel de l'Outaouais.

18 Les communications étaient donc intégrées au projet de la recherche-action. Nous pouvons même parler d'une vision « communicationnelle » du projet dans le sens d'une vision où les communications jouent un rôle tellement essentiel qu'elles constituent la 
charpente du projet. L'idée de base des initiateurs du projet a été de créer un espace chaleureux d'échange entre représentant-e-s des communautés culturelles et représentant-e-s municipaux-ales. Ils étaient convaincus que les élu-e-s municipaux-ales ne réagiraient favorablement que si l'ambiance était non-conflictuelle. L'idée a été de permettre un échange où les élu-e-s auraient la possibilité de se rendre compte que les représentant-e-s des communautés culturelles étaient des résident-e-s (et électeurs électrices) bien intégré-e-s dans leurs villes et qui voulaient essentiellement les mêmes choses que tou-te-s les résident-e-s. De cette façon, leur perception d'une hétérogénéité croissante serait positive et pourrait amener, espéraient les initiateurs du projet, une prise de conscience que la municipalité devrait jouer un rôle dans le domaine des relations interculturelles. Le succès du projet dépendait donc de l'établissement des contacts interpersonnels harmonieux.

La direction du projet a été réalisée par une chercheuse attachée au Bureau des services à la collectivité de l'Université du Québec à Hull, à la suite d'un protocole d'entente entre le Conseil et l'Université. Les objectifs de la recherche-action ont été fixés :

20 a) Informer les élus municipaux de villes d'Aylmer, de Hull et de Gatineau sur la réalité pluraliste de leur quartier respectif.

b) Susciter une concentration active entre les intervenants concernés en vue de faciliter l'intégration des nouveaux arrivants et de favoriser le rapprochement entre les différentes communautés.

c) Encourager l'ensemble des administrations municipales à se doter d'un plan d'action concerté relatif à la problématique de l'accueil des immigrants et y affecter les ressources nécessaires. (Denyse Lefebvre, Rapport de recherche sur la recherche-action sur les municipalités au coeur du défi interculturel, Université du Québec à Hull, 1993, Annexe C)

La recherche-action se déroulait en deux étapes. La première consistait en une série de réunions, par municipalités, entre les élu-e-s municipaux-ales et les représentantes des communautés culturelles. Il y a eu cinq réunions, deux à Hull, deux à Gatineau et une seule à Aylmer. Dans la deuxième étape de la recherche, un colloque régional a été organisé, réunissant tous les participant-e-s de la première étape et visant également une participation du public. L'objectif de la première série des réunions a été de soulever des thèmes de discussion ; thèmes qui pourraient être approfondis lors du colloque régional.

En préparant les premières réunions, une des décisions les plus importantes a été celle de choisir les informations qui devraient être fournies aux participant-e-s pour appuyer le contact interpersonnel. Après de longues discussions, quatre types d'informations ont été données : des données démographiques sur la population immigrante de chaque quartier, des résultats d'une pré-enquête auprès des membres des communautés culturelles sur leurs perceptions de leur municipalité, des exemples d'initiatives municipales au Canada et au Québec et finalement une synthèse des informations auprès des fonctionnaires des trois villes sur les activités existant dans le domaine interculturel

Ces informations se sont avérées beaucoup moins importantes pour les élu-e-s que la communication interpersonnelle. Les données par quartier ont été scrutées et les informations sur les activités existantes vérifiées mais, de façon générale, les élu-e-s ont donné plus de poids aux informations "vécues" qu'aux informations "scientifiques". Cette constatation ne veut pas dire, néanmoins, que ces informations ont été inutiles; les fonctionnaires qui assistaient aux réunions en ont pris note et, plus généralement, l'existence de ces informations a rassuré les participantes sur le sérieux du projet. 
26 Les réunions, pour la plupart, se déroulaient comme les initiateurs du projet les ont conçues. Le ton a été harmonieux et positif, dominé par le thème de l'importance de mieux se connaître. L'idée que le problème de fond était la faible quantité des communications entre les élues municipaux-ales et les représentant-e-s des communautés culturelles (et plus largement le peu de communications entre la société d'accueil et les membres des communautés culturelles) a dominé les rencontres et canalisé les recommandations. Toutes sortes d'idées ont été soulevées mais celles qui correspondaient au modèle « communicationnel » ont été favorisées. Nous reprenons en annexe la liste complète des recommandations du rapport final, pour souligner la centralité des questions définies en termes de communication.

\section{La conception des communications véhiculée dans la recherche-action}

27 Les communications et les problèmes reliés au manque de communication furent, comme nous l'avons décrit, l'un des éléments clé de la recherche-action. Le but de cette section est de décrire de quelle façon les communications ont été envisagées et comment les participant-e-s les ont conçues. La caractéristique principale de la vision des communications véhiculée dans la recherche-action était la primauté des contacts interpersonnels. Le modèle mettait beaucoup d'importance sur les individus et sur le caractère personnel, individuel et volontariste de la communication interpersonnelle. L'un des conseillers reflétait bien cette vision interpersonnelle quand il a dit : «Il faut se parler, il faut mieux se connaitre, il faut se dire des deux côtés qui on est. Si le monde se connaissait mieux, il n'y aurait pas de problèmes ».

Nous comprenons mieux l'utilisation de cette vision « communicationnelle » en regardant séparément pourquoi les représentant-e-s municipaux-ales et les représentant-e-s des communautés culturelles l'ont trouvé intéressante. Du côté du monde municipal ce discours reprend bien une vision de l'égalité d'opportunité. Il n'y a pas de structures sociales inégales; il y a simplement des gens qui devraient se parler. Il n'y a pas de question de racisme ou de discrimination raciale - il y a des gens qui doivent se parler. D'ailleurs, la responsabilité de la situation actuelle est tout à fait partagée par les différents partenaires - «il faut se dire des deux côtés qui on est». La population immigrante a une responsabilité et pas seulement les élu-e-s municipaux-ales. On parle des personnes égales mais qui n'ont pas échangé suffisamment d'informations. Cette vision laisse peu de place pour des interventions proactives de la part des autorités municipales - les problèmes se situent quasiment à l'extérieur de la sphère politique. En même temps, ce discours s'adapte très bien à la réalité électorale. Des contacts personnels sont essentiels au système politique - les conseillers sont donc intéressés à rencontrer des gens de leur quartier car ils se voient d'abord et avant tout comme les porte-parole légitimes de leurs citoyens.

30 Si nous pouvons comprendre aisément pourquoi ce discours plait aux responsables municipaux, il est moins évident de comprendre pourquoi les représentant-e-s des communautés culturelles l'ont utilisé. Évincer les rapports inégaux de pouvoir, évincer la question du racisme et de la discrimination raciale - quel avantage y-a-t-il ? Mais, en réalité, ce discours « interpersonnel » a dominé les interventions des représentant-e-s des 
communautés culturelles presque autant que celles du monde municipal. Premièrement, la recherche-action a véritablement débouché sur des contacts positifs entre l'appareil municipal et la population immigrante et pour la plupart des représentant-e-s des communautés culturelles il a été intéressant et enrichissant d'avoir des contacts amicaux avec des élus municipaux.

31 En plus, les représentant-e-s des communautés culturelles étaient, dans la majorité des cas, des personnalités fortement impliquées dans leur milieu et la recherche-action leur offrait la possibilité de gagner la reconnaissance des autorités municipales. Ces dernières ont d'ailleurs fait appel à leur leadership en leur confiant la responsabilité de retransmettre l'information aux autres membres de leurs communautés culturelles et de recueillir les commentaires de ceux-ci pour les transmettre aux municipalités. Il y avait donc une reconnaissance de l'importance des représentant-e-s des communautés culturelles en tant qu'agents de liaison entre les structures municipales et les communautés culturelles.

Nous verrons donc comment ce discours basé sur l'importance des contacts personnels se structure - il implique une vision des messages des municipalités vers les communautés culturelles et des messages des communautés culturelles vers les municipalités. A première vue, insister sur les contacts personnels semble suggérer un monde sans structure sociale ni institutionnelle, un monde où des individus égaux sont en interaction. Mais en réalité cette vision de l'importance des contacts personnels reflète une conception hiérarchisée - le gouvernement d'une part et les membres des communautés culturelles de l'autre. Les communications sont de deux ordres; descendante (municipalités vers les communautés culturelles) et ascendante (communautés culturelles vers les municipalités). Voyons comment les participant-e-s à la recherche-action concevaient ces deux formes de messages et quelles améliorations ils considéraient importantes d'envisager.

\section{Mécanismes de transmission d'informations de la municipalité vers les citoyens issus de communautés culturelles :}

Un des discours dominants de la recherche-action partait du fait que la population immigrante connaît très peu les services municipaux, qu'elle n'est donc pas portée à les utiliser et qu'elle demeure ainsi perpétuellement absente de cette institution. Selon cette logique, les communications sont perçues comme un moyen privilégié d'encourager la participation à la vie collective. Néanmoins, il est difficile de savoir exactement si ce manque de visibilité et d'accessibilité des services municipaux est causé par des faiblesses au niveau de la municipalité (au plan des relations publiques ou des services de communication) ou plutôt s'il était davantage le résultat des facteurs culturels liés à la réalité des communautés culturelles. Plusieurs intervenant-e-s, tant municipaux qu'issus des communautés culturelles semblaient vouloir mettre l'explication du côté des communautés culturelles en déclarant que les publications municipales étaient sans reproche dans la mesure où elles transmettaient des informations de qualité en quantité appropriée. Par contre, ces participant-e-s insistaient sur les obstacles à la réception efficace de ces informations chez les membres des communautés culturelles. 


\section{Les obstacles à la réception des communications de la municipalité :}

\section{La peur et la crainte}

D'abord, il y a la peur des institutions politiques. Pour certains immigrant-e-s et réfugiée-s, les appareils bureaucratiques font l'objet de méfiance. Certains néo-Québécois et néoQuébécoises ont quitté des pays où les autorités politiques, militaires et policières détenaient un pouvoir absolu. Dans de telles circonstances, il est très compréhensible que toute communication venant d'un gouvernement soit traitée avec grande appréhension.

\section{La tradition orale versus la tradition écrite}

Pour les membres de certaines communautés culturelles, l'information importante concernant la vie publique est traditionnellement transmise de bouche à oreille. Or le système canadien repose sur une culture de l'écriture ce qui ne correspond pas du tout aux habitudes de ces groupes. Pour ces gens, tous les beaux dépliants produits par les municipalités n'ont aucune valeur, car ils ne respectent pas la culture orale des récipiendaires des messages. Les participant-e-s sensibles à cette question favoriseraient davantage des messages publicitaires télévisuels ou radiophoniques plutôt que les documents écrits.

\section{Les facteurs linguistiques et culturels}

Un des plus gros obstacles demeure la barrière linguistique qui, souvent, affecte davantage les personnes du troisième âge plus que tout autre groupe. Le recours à des documents municipaux dans plusieurs langues n'est pas encore fréquent dans les municipalités étudiées. D'ailleurs ce blocage linguistique s'ajoute souvent à des barrières plus culturelles. Chez certaines communautés, par exemple, la participation des femmes à la vie publique est limitée par les traditions culturelles. Même les bons documents, adaptés à la langue de la communauté culturelle ne seront pas reçus ni compris à cause d'un manque de sensibilité culturelle.

\section{Mécanismes de communication des citoyens issus de communautés culturelles vers la municipalité}

Les discussions sur les mécanismes de communication portent également beaucoup sur les moyens de recueillir les réactions populaires car « l'effort doit venir des deux côtés ». De façon générale, outre les élections, il existe très peu de mécanismes permettant aux citoyens d'acheminer leurs préoccupations aux autorités municipales afin d'influencer l'activité politique de la ville. Les mécanismes qui existent sont relativement peu structurés (contacts au téléphone ou en personne avec les services municipaux) et donc leur efficacité dépend souvent de l'insertion sociale de la personne. Dans le cas de la population immigrante, les réseaux de contacts personnels ne sont pas encore solidement établis (comparativement, par exemple, aux groupes de gens d'affaires ou à certains clubs sociaux). Deux options s'imposent donc: soit les représentants des communautés 
culturelles se conforment aux mécanismes déjà en existence ou ils travaillent à leurs besoins spécifiques en assurant un lien plus constant entre eux et les élus municipaux.

Cette volonté d'élargir les réseaux de communication se manifeste très concrètement dans certaines des recommandations de la recherche-action. En effet, il fut beaucoup question, lors des rencontres et du colloque d'établir un réseau permanent de communication entre les municipalités et les communautés culturelles afin d'assurer un flux constant d'informations. Que ce soit en rapport avec les activités culturelles, sportives, sociales ou encore avec les offres d'emplois, l'idée d'un lien plus organisé et plus constant entre les communautés culturelles et les municipalités semblent offrir des avantages. La recommandation qui reflète le mieux cette approche est celle proposant la création d'un comité interculturel aviser auprès des municipalités sur lequel siégeraient des élu-e-s, des représentant-e-s de l'administration municipale et des représentantes communautés culturelles. L'idée de la création d'un canal de communication dominé par des échanges directs combine une certaine volonté de formalisation avec la vision de l'importance des contacts interpersonnels.

\section{L'importance des communications}

En conclusion, tentons d'expliquer la centralité de cette vision «communicationnelle » des relations interculturelles que nous avons observée lors de la recherche-action auprès des municipalités de l'Outaouais québécois.

Un premier élément de la réponse vient du fait que la région de l'Outaouais n'a pas encore connu d'incidents raciaux graves et que, donc, l'argumentation pour une intervention gouvernementale a été formulée plus en terme de prévention qu'en terme de réparation. L'établissement de meilleures communications entre les différents groupes de la population semble particulièrement bien adapté à cette vision du travail préventif. La grande majorité des participant-e-s aux rencontres acceptait cette définition de la situation actuelle: une situation où il n'y avait pas de conflits et un ou une des représentantes des communautés culturelles soulignait que la situation actuelle comportait très occasionnellement des inégalités mais que l'expression de ces inégalités pouvait faire avancer le dossier. Bref, l'importance des communications cadrait bien avec la perception de la situation interculturelle qui était largement acceptée par l'ensemble des participant-e-s.

41 Un deuxième élément de réponse vient de la vision politique des acteurs municipaux. Comme nous l'avons constaté, les élu-e-s municipaux-ales véhiculent, en général, une vision libérale du fonctionnement du système politique - un système où les citoyens ont tous à peu près les mêmes possibilités d'avoir des services. Les améliorations au fonctionnement visent donc à assurer cette égalité et ceci peut se réaliser en favorisant les communications. De bonnes communications peuvent résoudre les problèmes de chances inégales et donc justifient la vision de la politique municipale des élu-e-s municipaux-ales. Les représentant-e-s municipaux-ales étaient donc à l'aise avec le modèle proposé et, par conséquent, prêts à accepter le déroulement de la rechercheaction. Cette acceptation était nécessaire à la mise sur pied du projet étant donné que celui-ci consistait à stimuler une activité par les municipalités. Le modèle « communicationnel » correspondait bien à cette dimension du projet. 
42 La centralité des communications était inscrite dans la recherche-action dès sa formulation. On visait des échanges amicaux où la chaleur des contacts interpersonnels pourrait convaincre les municipalités qu'elles avaient plus à gagner qu'à perdre avec une activité accrue dans le domaine des relations interculturelles. L'importance des communications est confirmée par la recherche-action qui démontre qu'une communauté ne peut fonctionner sans un circuit d'échanges qui irrigue les deux pôles d'interaction.

\section{Annexe : Recommandations}

\section{Actions d'ordre général}

43 Voici une liste d'actions souhaitables qui se dégagent des discussions que les participants ont eues à l'occasion du colloque et des rencontres par ville.

44 - L'élaboration d'une politique globale proactive, coordonnée et structurée qui permettait de faire connaître les services existants, de créer des partenariats et de confirmer le leadership des villes dans le dossier.

45 - La prise en charge des éléments de la politique sociale notamment celui relié à l'intégration des communautés culturelles.

46 - Créer un comité interculturel avisé auprès de la municipalité sur lequel siégeraient des élus, des représentants de l'administration municipale et des communautés culturelles.

47 - Créer des occasions pour que les communautés culturelles puissent échanger avec les résidents de souche pour qu'ensemble ils puissent apprécier l'autre culture et vivre en harmonie.

48 - Examiner comment l'administration municipale s'adapte à cette nouvelle réalité sociale et recommander un plan d'action.

49 - Identifier les besoins spécifiques des communautés culturelles en fonction de leur propre diversité.

50 - Favoriser l'action dans les domaines du développement économiques, des loisirs et des activités sociales, de la sécurité publique, du logement et de l'urbanisme, de l'embauche et de la communication.

51 - Fournir aux employés municipaux un programme de formation en relations interculturelles.

\section{Actions spécifiques}

\section{Logement et urbanisme}

52 - Clarifier la division des responsabilités entre la SHQ et le palier local.

53 - Adapter les politiques pertinentes.

54 - Intégrer le phénomène de l'immigration dans le plan d'urbanisme.

55 - Innover au niveau de la fiducie foncière communautaire.

56 - Négocier avec les propriétaires, lors de l'approbation des plans, l'inclusion d'un certain nombre de logements sociaux. 
57 - Examiner comment utiliser le domaine du logement pour attirer d'autres immigrants dans l'Outaouais et les garder.

58 - Besoin d'un meilleur système d'information dans le domaine.

59 - Faciliter l'accès au logement par un travail autant auprès des communautés culturelles que des associations de propriétaires.

60 - Identifier une personne ou un lieu pour l'accueil.

61 - Établir un centre d'accueil qui servirait de lieu d'hébergement pour les premiers jours passés dans la région de résidence surtout en ce qui concerne les réfugiés.

\section{Loisirs et culture}

62 - Favoriser les échanges culturels entre les Québécois de souche et les communautés culturelles.

63 - Organiser des activités ethnoculturelles dans les quartiers.

64 - Favoriser les activités sportives chez les enfants pour intégrer les parents via cette activité.

65 - Utiliser les bibliothèques pour informer les groupes ethniques.

66 - Encourager les prêts de livres « inter-municipalité ».

67 - Identifier les besoins des immigrants en matière de loisirs.

68 - Revoir les modes de consultation traditionnels. Repenser les mécanismes actuels relativement aux critères de reconnaissance des organismes régionaux.

69 - Organiser une rencontre des villes pour revoir ce que chacune a accompli pour répondre aux besoins des différentes cultures.

70 - Examiner si la promotion des loisirs rejoint les immigrants en tenant compte que, pour certains cultures, les messages oraux sont plus efficaces que les messages écrits.

\section{Développement économique}

71 - Besoin d'une attitude dynamique et proactive face à l'immigration économique.

72 - S'assurer que les politiques de développement et de promotion économiques des municipalités tiennent compte de l'immigration économique.

73 - Déterminer les besoins en matière de développement économique (types de secteur à développer, types d'emploi).

74 - Définir des secteurs prioritaires qui pourraient bénéficier des retombées de l'immigration économique (e.g. haute technologie).

75 - Définir le profil des immigrants (gens d'affaires, travailleurs spécialisés) en fonction des secteurs retenus.

76 - Favoriser la concertation entre les villes dans une perspective d'élaboration d'un plan d'action régional.

77 - Adapter les outils de promotion à la dimension de l'immigration économique (Ne pas valoriser uniquement l'aspect touristique mais aussi l'aspect économique).

78 - Assurer un service de liaison entre les municipalités, les intervenants économiques et les nouveaux arrivants afin d'aider ces derniers à réaliser leurs projets d'affaires. 


\section{Ressources humaines et politiques d'embauche}

79 - Élaborer une politique d'embauché qui comprend un programme d'équité en matière d'emploi pour les communautés culturelles.

80 - Établir un réseau permanent de communication entre la Ville et les communautés culturelles afin de diffuser les offres d'emploi en-dehors des circuits traditionnels.

81 - Évaluer les procédures d'embauché et l'ensemble du système des ressources humaines afin de déterminer les formes de discrimination qu'ils peuvent entretenir ou qui leur sont inhérentes.

\section{Communication et relations interculturelles}

82 - L'engagement formel des municipalités à vouloir rejoindre les différentes communautés (Résolution du Conseil, directives formelles aux divers services).

83 - Identifier des moyens de communication adéquats pour communiquer avec les communautés culturelles (Bulletin, agent de relation) et utiliser les moyens de communication existant dans les communautés.

84 - Obtenir la participation des membres des communautés culturelles.

85 - Identifier et utiliser les organismes ou institutions qui peuvent aider les villes dans l'atteinte de leurs objectifs de communication.

86 - Établir un système d'évaluation des actions entreprises par les villes et les communautés culturelles.

87 - Prévoir un budget pour encourager la communication et les activités «villescommunautés ».

\section{BIBLIOGRAPHIE}

Bonneau, Micheline et Pierre André Tremblay Immigration et région : nouveaux enjeux, nouvelles perspectives, Chicoutimi, Chaire d'enseignement et de recherche interethniques et interculturelles, 1993.

Folco, Raymonde, La régionalisation de l'immigration : les services municipaux, Allocution prononcée par la présidente du Conseil des communautés culturelles et de l'immigration, 1993.

Laroche, Gabriel, « Les facteurs de décision dans le choix par les immigrants d'une région de résidence : éléments d'éclairage », dans Micheline Bonneau et Pierre-André Tremblay, Immigration et région, op. cit, 1993.

Manegre, Jean-François, « La régionalisation de l'immigration du rêve à la réalité... », dans Micheline Bonneau et Pierre-André Tremblay, Immigration et région, op. cit, 1993. 
Ministère des affaires municipales, Vers un nouvel équilibre: Partage des responsabilités Québecmunicipalités, Québec, 1990.

Secrétariat régional de la concertation de l'Outaouais, Actes du colloque national sur la régionalisation de l'immigration au Québec, Hull, 1992.

\section{RÉSUMÉS}

Ce texte analyse l'activité des municipalités dans le domaine des relations interculturelles. Cette analyse se fait par une ététude de cas d'une expérience de recherche-action effectuée par le Conseil interculturel de l'Outaouais auprès des trois plus grandes municipalités de l'Outaouais québécois. Le but de l'étude de cas est d'examiner le rôle joué par les communications dans les relations entre les communautés culturelles et les municipalités et de tenter d'expliquer leur importance. Après une mise en contexte par rapport au système municipal québécois en général et aux trois municipalités de l'Outaouais, l'étude examine le fonctionnement de la rechercheaction et la centrante des communications dans ce projet. L'étude conclut à l'importance des communications de la formulation même de la recherche-action mais aussi de la conception qu'ont les acteurs de la façon dont une communauté doit fonctionner.

This article analyzes municipal activity in the area of intercultural relations. This is done by means of a case study of an action-research project done by the Outaouais Intercultural Council in conjunction with the three largest municipalities in the Outaouais region of Quebec. The objective of the case study is to examine the role played by communications in the relations between the cultural communities and the municipalities and to attept to explain their importance. After setting the context in terms of the Québec municpal System in general and the three Outaouais municipalities, the study examines the way the action-research unfolded and the central role played by communications. The conclusions of the study are that the importance of communication comes from the very way in which the action-research was formulated but also from the conception held by the actors involved of how a community should fonction.

\section{AUTEURS}

\section{CAROLINE ANDREW}

Caroline Andrew est professeur au Département de science politique à l'Université d'Ottawa. Elle s'intéresse à la politique municipale au Canada ainsi qu'aux politiques de développement urbain. Elle mène présentement un projet de recherche portant sur les politiques sociales dans les municipalités canadiennes tout particulièrement dans deux domaines ; les relations interculturelles et les questions intéressant les femmes.

\section{LOUISE LEGAULT}

Louise Légault a complété ses études de premier cycle au Département de science politique à l'Université d'Ottawa et elle y est actuellement inscrite au programme de deuxième cycle au Département de sociologie. Elle a été assitante pour le projet de recherche portant sur l'activité des municipalités dans le domaine des relations interculturelles et elle travaille présentement comme assistante pour un projet de recherche au Département de Communication de l'Université d'Ottawa portant sur les nouvelles technologies et la vie quotidienne. 